\title{
High expression of stromal signatures correlated with macrophage infiltration, angiogenesis and poor prognosis in glioma microenvironment.
}

\author{
Yixin Tian ${ }^{1,2}$, Yiquan Ke ${ }^{\text {Corresp., 1, } 2}$, Yanxia Ma ${ }^{\text {Corresp. 1, } 2}$ \\ ${ }^{1}$ Department of Neurosurgery, Zhujiang Hospital, Southern Medical University, The National Key Clinical Specialty, The Engineering Technology Research \\ Center of Education Ministry of China, Guangdong Provincial Key Laboratory on Brain Function Repair and Regeneration, Guangzhou, China \\ 2 Key Laboratory of Mental Health of the Ministry of Education, Guangdong-Hong Kong-Macao Greater Bay Area Center for Brain Science and Brain- \\ Inspired Intelligence,Southern Medical University, Guangzhou, Chian \\ Corresponding Authors: Yiquan Ke, Yanxia Ma \\ Email address: kyquan@smu.edu.cn, laoma888xin@sina.com
}

Glioma is one of the most fatal tumors in central nervous system. Previous studies gradually revealed the association between tumor microenvironment and the prognosis of gliomas patients. However, the correlation between tumor-infiltrating immune cell and stromal signatures are unknown. In our study, we obtained gliomas samples from CGGA and TCGA. The landscape of tumor infiltrating immune cell subtypes in gliomas was calculated by CIBERSORT. As a result, we found high infiltration of macrophages was correlated with poor outcome $(P<0.05)$. Then functional enrichment analysis of high/low macrophage-infiltrating groups was performed by GSEA. The results showed 3 gene sets includes 102 core genes about angiogenesis were detected in high macrophage-infiltrating group. Next we constructed PPI network and analyzed prognostic value of 102 core genes, we found that 5 stromal signatures indicated poor prognosis which including HSPG2, FOXF1, KDR, COL3A1, SRPX2 ( $\mathrm{P}<0.05)$. 5 stromal signatures were adopted to construct a classifier. And the classifier showed powerful predictive ability $(A \cup C=0.748)$. Patients with high risk score held poor survival. Finally, we validated this classifier in TCGA and the result was consistent with CGGA. Our investigation of tumor microenvironment in gliomas may stimulate the new strategy in immunotherapy. And 5 stromal signature correlated with poor prognosis also provide a strong predator of gliomas patient outcome. 
1 High expression of stromal signatures correlated with

2 macrophage infiltration, angiogenesis and poor 3 prognosis in glioma microenvironment.

4

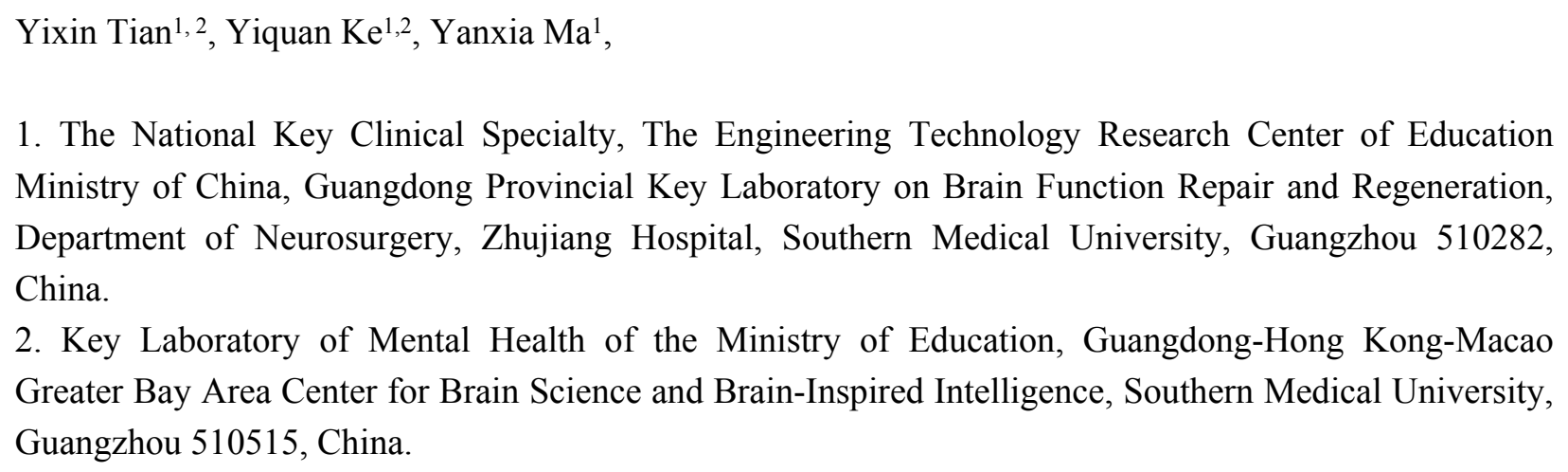




\section{Abstract}

Glioma is one of the most fatal tumors in central nervous system. Previous studies gradually revealed the association between tumor microenvironment and the prognosis of gliomas patients. However, the correlation between tumor-infiltrating immune cell and stromal signatures are unknown. In our study, we obtained gliomas samples from CGGA and TCGA. The landscape of tumor infiltrating immune cell subtypes in gliomas was calculated by CIBERSORT. As a result, we found high infiltration of macrophages was correlated with poor outcome $(\mathrm{P}<0.05)$. Then functional enrichment analysis of high/low macrophage-infiltrating groups was performed by GSEA. The results showed 3 gene sets includes 102 core genes about angiogenesis were detected in high macrophage-infiltrating group. Next we constructed PPI network and analyzed prognostic value of 102 core genes, we found that 5 stromal signatures indicated poor prognosis which including HSPG2, FOXF1, KDR, COL3A1, SRPX2 ( $<<0.05)$. 5 stromal signatures were adopted to construct a classifier. And the classifier showed powerful predictive ability $(\mathrm{AUC}=0.748)$. Patients with high risk score held poor survival. Finally, we validated this classifier in TCGA and the result was consistent with CGGA. Our investigation of tumor microenvironment in gliomas may stimulate the new strategy in immunotherapy. And 5 stromal signature correlated with poor prognosis also provide a strong predator of gliomas patient outcome.

\section{Introduction}

Glioma is the most deadly central nervous system (CNS) tumor. Patients with gliomas always show poor outcome, and their median overall survival remains from 14.6-16.8 months. The prognosis of gliomas patients is correlated with tumor subtype, age and sex(SMJ et al. 2018). Based on tumor morphology and molecular alterations, the WHO updated a new classification system in 2016 (AM et al. 2019). Gliomas are classified based on the absence or presence of mutations in IDH and the absence or presence of 1p/19q chromosomal co-deletion(M et al. 2018):IDH-mutant, 1p/19q-co-deleted present a benign prognosis; IDH-mutant, non-1p/19q-codeleted have intermediate survival outcomes; and IDHwild-type have an unfavorable prognosis. The poor prognosis of gliomas has stimulated the discovery of new treatment strategies such as immunotherapy(HK et al. 2017; ML et al. 2018). However, Gliomas presents significant resistance to immunotherapy. Resistance of innate immunity prevents the tumor from an immune response, resistance of adaptive immunity deactivates tumor-infiltrating immune cells(CM et al. 2019). Thus, in order to carry out further study of immunotherapy, it's vital to figure out the tumor microenvironment in gliomas. Previous study integrated several tumor microenvironment related genes based on TCGA database applied by ESTIMATE algorithmbased immune scores(B et al. 2018). Even so, the correlation between tumor-infiltrating immune cells and stromal signatures in gliomas are still unknown.

The Chinese Gliomas Genome Atlas (CGGA) is a database which contains 2,000 gliomas samples from Chinese cohorts. And this database was finished a large scale update in July 2019, 693 mRNA 
sequencings and matched clinical data were provided, which includes: histology, WHO grades, age, gender, chemotherapy, radiotherapy, overall survival. CIBERSORT is a computational method for quantifying 22 immune cell subtypes fractions from gene expression profile such as microarray or RNAseq(B et al. 2018). Flow cytometry verified this computational method and several studies showed the landscape of tumor-infiltrating immune cells can be accurately determined by this method in various malignant tumors such as colon cancer, breast cancer, lung cancer and prostate cancer(E et al. 2019; HR et al. 2016; $X$ et al. 2017).

In this study, we extracted 693 RNA-seq and matched clinical data from CGGA database. Then we analyzed the differences of 22 immune cell subtypes between normal brain tissue and gliomas, and revealed their prognosis value respectively. We also compared immune cell subtypes among different phenotypes such as: histology, WHO grades, age, gender, chemotherapy, radiotherapy, IDH and 1p/19q chromosomal co-deletion. Next, we identified 5 stromal signatures with high macrophages infiltration and angiogenesis which significantly contribute to prognosis by functional enrichment analysis and proteinprotein interaction networks. And the stromal signatures were adopted to construct a classifier. The classifier showed powerful predictive ability $(\mathrm{AUC}=0.748$ ). Patients with high risk score held poor survival. Finally, we validated this classifier in TCGA and the result was consistent with CGGA. The results provided a comprehensive view of tumor microenvironment and may stimulate new strategy of immunotherapy in Chinese gliomas patients.

\section{Methods}

\section{Collecting RNA-seq and matched clinical data from CGGA and TCGA.}

We built two independent cohorts for training set and testing set from CGGA and TCGA. The gene expression profiles were downloaded from The Chinese Gliomas Genome Atlas (CGGA http://www.cgga.org.cn/). 695 RNA-seq data of Chinese gliomas patients were obtained from CGGA database. And a series of measures were taken: (1) Genes with a variance of 0 will be filtered out. (2) Complete follow-up information and samples with a follow-up time $>30$ days will be served. Finally, 693 samples meeting the inclusion criteria were included. And each patient matched specific clinical data, which includes: histology, WHO grades, age, gender, chemotherapy, radiotherapy, survival status, survival duration in days. To build the testing cohort, The RNA-seq FPKM of gliomas including corresponding outcome data were downloaded from The Cancer Genome Atlas (TCGA; https://cancergenome.nih.gov/). The following measures were taken: (1) The IDs were annotated on the basis of hg38 reference genome. (2)Genes with a variance of 0 will be filtered out. (3)For the same gene corresponding to multiple IDs or a patient with multiple tumor samples, the average will be taken. (4) Samples with a follow-up time $>30$ days were remained. Eventually, a total of 668 TCGA samples fulfilled our criteria. The details of patients information was showed in Table 1.

\section{Analysis of tumor-infiltrating immune cells}

CIBERSORT is a computational method for quantifying immune cell subtypes fractions from normalized gene expression profiles based on deconvolution algorithm. The leukocyte gene signature matrix (LM22) is generated for the calculation of 22 human hematopoietic subsets. LM22 has been 
verified on Affymetrix HGU133 and Illumina Beadchip platforms. In our study, we used LM22 gene file to calculate 22 immune cells subtypes of 693 gliomas patients. These immune cell subtypes included CD8+ $\mathrm{T}$ cells, naïve CD4+ $\mathrm{T}$ cells, resting CD4+ memory $\mathrm{T}$ cells, activated CD4+ memory $\mathrm{T}$ cells, follicular helper T cells (Tfh), Tregs, $\gamma \delta \mathrm{T}$ cells, naïve B cells, memory B cells, plasma cells, resting NK cells, activated NK cells, resting NK cells, activated NK cells, resting dendritic cells, activated dendritic cells, Monocytes, Eosinophils, Neutrophils M0 macrophages, M1 macrophages, M2 macrophages, resting mast cells and activated mast cells,. And CIBERSORT p-value $<0.05$ was demanded. The median cutoff was determined to separate the patients into the high-infiltrating or low-infiltrating groups.

\section{Statistical analysis}

The differences of immune cell subtypes between normal brain tissues and gliomas tissues were assessed using the unpaired $t$-test, and the differences of tumor-infiltrating immune cells among different phenotypes were performed by t-test and one One-way analysis of variance (ANOVA). Kaplan-Meier curves were used to perform the correlations of 22 immune cell subtypes or gene signature and corresponding clinical follow-ups (log-rank test), and high versus low groups all based on median cut off. Statistical analyses were calculated by SPSS statistical package software or GraphPad Prism. P values $<0.05$ were considered significant.

\section{Enrichment analysis}

Functional enrichment analyses of tumor-infiltrating immune cells were performed by GSEA (Gene Set Enrichment Analysis). We analyzed via GSEA v4.0.3 for Windows (http://software.broadinstitute.org/gsea/index.jsp) (Subramanian et al. 2005). GO categories (M et al. 2000) include biological processes (BP), molecular functions (MF), or cellular components (CC) and KEGG (Kyoto Encyclopedia of Genes and Genomes) (https://www.kegg.jp/) pathway were analyzed by GSEA(J et al. 2019). FDR $<0.1$ and $\mathrm{P}<0.01$ were considered significant. Protein-protein interaction (PPI) networks were constructed by the STRING tool (https://string-db.org/) and analyzed by Cytoscape (P et al. 2003). PPI network was used to identify the hub gene. And the gene set which contain 149 stromal signatures was generated in both ESTIMATE and MSigDB.

\section{Construction of classifier}

To generate and optimize the prognostic classifier, the multivariate Cox regression analysis was performed in CGGA and TCGA cohort. We calculated the risk score of each sample based on the multivariate COX coefficient, and the low/high risk groups were defined according to the median cutoff risk score. The receiver operator characteristics (ROC) curve analysis was applied to assess the classifier's ability to distinguish samples with a high or low risk score, and it was draw by survivalROC package. The area under the curve (AUC) of the ROC curve was calculated and compared to examine the performance of the classifier in both training and testing cohorts. The median risk score (RS) was determined to separate the genes into the high-risk or low-risk groups. KM curves and independent testify were performed by survival package to assess the effective of classifier in gliomas patients (log-rank test). All analysis were carried out by $\mathrm{R}$ version 3.6.1 and corresponding packages. 


\section{Proteomics and histology.}

To verify the infiltration of M2 macrophage in glioma patients, we downloaded proteomics data of 110 glioma patients from The National Cancer Institute's Clinical Proteomic Tumor Analysis Consortium (CPTAC; https://cptac-data-portal.georgetown.edu/). And the histological level research of glioma patients was performed in the human protein atlas (https://www.proteinatlas.org/).

\section{Result}

\section{The landscape of tumor infiltrating immune cell subtypes in gliomas}

Based on the 693 RNA-seq from CGGA database, the different infiltration of 22 immune cell subtypes between normal brain tissue and gliomas were analyzed by $t$-test. The fraction of M0 macrophages, M2 macrophages, resting NK cells, plasma cells, CD8+ T cells was significantly higher in gliomas tissue than normal brain tissue (Fig. 1A). On the contrary, the fraction of naïve B cells, resting dendritic cells, Eosinophils, Neutrophils, M1 macrophages, activated mast cells, follicular helper T cells (Tfh), $\gamma \delta$ T cells and resting CD4+ memory T cells was significantly lower in gliomas tissue than normal brain tissue (Fig. 1B).

A total of 9 clinical parameters were analyzed, which includes: age, gender, histology, WHO grades, primary or recurrence, chemotherapy, radiotherapy, mutations in IDH and $1 \mathrm{p} / 19 \mathrm{q}$ co-deletion. As a result, gender, primary or recurrence, chemotherapy showed no significant difference of immune cells. Monocytes were decreased in elderly patients (Fig2. A). M0 macrophages, Tregs and activated dendritic cells were increased in high grade gliomas, whereas monocytes were decreased (Fig2.B). The fraction of M0 macrophages, Tregs and $\gamma \delta \mathrm{T}$ cells was higher in glioblastoma (GBM) than astrocytoma (AOA), whereas monocytes and activated mast cells was lower (Fig2.C). The fraction of activated mast cells, monocytes and resting CD4+ memory $\mathrm{T}$ cells was higher in IDH mutant than wildtype, while M0 macrophages, Tregs, $\gamma \delta \mathrm{T}$ cells and Tfh was lower (Fig2.D). Tregs were increased in 1p/19q co-deletion, whereas naïve CD4+ T cells were decreased (Fig2.E).

\section{Prognostic value of tumor-infiltrating immune cells in gliomas}

In this study, we divided all 22 immune cell subtypes into high/low infiltration group based on median cutoff. And we analyzed the correlation between 22 immune cell subtypes and patients prognosis by generating Kaplan-Meier survival curve. Result showed that high infiltration of M0 macrophages $(\mathrm{P}=0.0104)$ and resting $\mathrm{CD} 4+$ memory $\mathrm{T}$ cells $(\mathrm{P}=0.027)$ had an unfavorable prognosis. (Fig3.A, $\mathrm{B})$ Based on the previous studies, M2 macrophages showed pro-tumor function as well as M1 macrophages showed anti-tumor function in gliomas. We constructed a macrophage score: (M0+M2)/M1. And results showed gliomas patients with high macrophage score correlated with poor outcome. (Fig3.C)

\section{Functional enrichment analysis of gene signature of macrophages}

As the infiltration of macrophages play a significant role in glioma patients' outcomes, we further investigated the potential mechanism and gene signature. First, we divided gliomas patients into high/low macrophage-infiltrating groups. Functional enrichment analysis was performed by GSEA (Gene Set Enrichment Analysis). GO categories include biological processes (BP), molecular functions (MF), 
198

199

200

201

202

203

204

205

206

207

208

209

210

211

212

213

214

215

216

217

218

219

220

221

222

223

224

225

226

227

228

229

230

231

232

233

234

235

236

237

238

cellular components (CC) and KEGG (Kyoto Encyclopedia of Genes and Genomes) pathway were analyzed. As a result, a total of $5 \mathrm{GO}$ terms of biological process, $2 \mathrm{GO}$ terms of cellular component, 8 GO terms of molecular function and 4 KEGG pathway terms were identified to be significant (FDR $<0.1$ and $\mathrm{P}<0.001$ ) (Figure 4). Interestingly, the 3 enrichment $\mathrm{GO}$ terms with high macrophage infiltration were correlated with angiogenesis, so we obtained the 102 core gene signatures for next investigation.

\section{Investigation of stromal signatures correlated with macrophage infiltration and angiogenesis}

We built protein-protein interaction networks to further investigate the 102 core gene signatures which correlated with high macrophage infiltration and angiogenesis by STRING tool. Cytoscape and Cytohubba were used to analyze hub genes (Supplement figure 3). And the gene set which contain 149 stromal signatures was provided by ESTIMATE and MSigDB. Then, we compared 102 hub genes with 149 stromal signatures. Eventually we generated 5 stromal signatures including HSPG2, FOXF1, KDR, COL3A1, SRPX2 (Fig5.A). To investigate the prognostic value of 5 stromal signatures, we analyzed overall survival by Kaplan-Meier survival curves in CGGA database. The result show that 5 stromal signatures were significantly correlated with poor outcome $(\mathrm{P}<0.05)$ (Fig5.B-F).

\section{Construction and verification of prognostic classifier based on stromal signatures.}

According to univariate COX regressions, all of 5 stromal signatures were significantly correlated with poor outcome in gliomas patients (HR $>1$ ) (Fig6.A). We calculated the risk score of each sample based on the multivariate COX coefficient, and the low/high risk groups were defined according to the median cutoff risk score. Then, K-M plot showed patients with high risk score had lower survival than patient with low risk score $(\mathrm{P}<0.001)$ (Fig6.B, E). Next, we generated univariate and multivariate analyses which enrolled clinical features and stromal signatures in the overall set. The result showed that stromal signature classifier was an independent factor for gliomas patients (Fig6.C, D). Furthermore, we found all of 5 stromal signatures were correlated with WHO grades $(\mathrm{P}<0.05)$ (Fig6.F). As for histology, the expression of stromal signatures in glioblastoma was significantly higher than astrocytoma and oilgodendroglioma. The expression of COL3A1, HSPG2 and SRPX2 in patients with chemotherapy was higher than patients without chemotherapy. And the expression of COL3A1, KDR and SRPX2 was higher than patients with radiotherapy than patient without radiotherapy (Supplement Figure 1). Principal component analysis showed significant different between groups of low and high risk score (Fig6.G). A receiver operating characteristic (ROC) curve was plotted to assess the effect of the classifier. The area under the curve (AUC) of the classifier was 0.748. Although stromal classifier was less efficient than the WHO grade, it was much superior to histological type (Fig6. H). To verify the classifier, we generated an independent cohort based on TCGA dataset. And the classifier also showed strong predictive ability in TCGA cohort (AUC=0.726) (Fig6. I).

\section{Verifying the infiltration of $\mathrm{M} 2$ macrophage in glioma patients.}

CD163 is the marker protein of M2 macrophage. By measuring the expression of CD163, the infiltration degree of M2 macrophage in glioma can be determined. We demonstrated high expression of CD163 in glioma patients through the CPTAC proteomics database. Meanwhile, we compared the immunohistochemistry of CD163 in normal brain tissue and glioma through the human protein atlas, and 
239

240

241

242

243

244

245

246

247

248

249

250

251

252

253

254

255

256

257

258

259

260

261

262

263

264

265

266

267

268

269

270

271

272

273

274

275

276

277

278

found that The expression of CD163 in glioma was significantly higher than that in normal brain tissue (Supplement figure 2).

\section{Discussion}

First of all, based on the RNA-seq data from CGGA and the CIBERSORT, we calculated 22 subtypes of tumor-infiltrating immune cells in gliomas. We comprehensively analyzed the tumor-infiltrating immune cells present in gliomas and revealed the prognosis value.

The landscape of tumor-infiltrating immune cells in gliomas as follows:

1. B cell lines: Naïve B cells can differentiate into antibody-secreting plasma cells after being stimulated by antigen(CK et al. 2016). Our study found that naïve B cell was a significant decrease in gliomas, meanwhile, the plasma cells were significant increased. It was illustrated that naïve B cell may be differentiated into plasma cells by gliomas antigens.

2. T cell lines: Tregs are correlated with unfavorable prognosis in the several kinds of tumor microenvironment (e.g. ovarian cancer, breast cancer, kidney cancer and pancreatic cancer)(R et al. 2017). Our results showed that Tregs were significantly increased in GBM and high WHO grade, which demonstrated Tregs may promote the development of gliomas(C et al. 2009; L \& SW 2012). Previous study showed that tumor-infiltrating Tfh cells have protective roles by suppressing lymphoid tumorpromoting effects in breast cancer and colorectal cancer(KI et al. 2018). Our results also shown that Tfh cells was significantly decrease in gliomas. Previous study showed neutrophils and eosinophils are implicated in almost every stage of oncogenesis and display both anti- and pro-tumor properties(CS et al. 2011; JR et al. 2018).

3. Myeloid cell lines: Our results showed that neutrophils and eosinophils were significantly decreased in gliomas, which indicated neutrophils and eosinophils may have negative correlation with gliomas. Mast cells play an important role in the growth of tumors( $\mathrm{J}$ et al. 2011). However, the contribution of mast cells in the microenvironment of solid malignancies remains controversial. Previous studies have illustrated that gliomas are associated with a profound accumulation of mast cells, and STAT5 play an important role on the recruitment of mast cells to gliomas(J et al. 2014). Our results showed that resting mast cells were increased in gliomas, whereas activated mast cells were significantly decreased in gliomas. In addition, the infiltration of activated mast cells of glioblastoma was prominently lower than astrocytoma. Consistent with previous study, gliomas promoted mast cells recruitment, but gliomas may also block resting mast cell activation. And glioblstoma may show stronger ability of preventing mast cell activation than astrocytoma. Dendritic cell vaccinations have emerged as newly strategies in the treatment of gliomas(AD et al. 2016; PY et al. 2019). Adjuvant dendritic cell-based immunotherapy for gliomas patients can induce long-term survival(TF et al. 2018). We found resting dendritic cells were significantly decreased in glioma, which was consistent with previous studies that dendritic cells recruitment correlated with favorable outcome. Previous study showed monocytes played an important role in cancer development and progression( $\mathrm{J}$ et al. 2011). And different monocytes displayed both anti- and pro-tumor function(M et al. 2013). Our results showed monocytes were significant decreased in high grade glioma and glioblastoma, which indicated stimulating monocytes recruitment may prevent progression of high grade gliomas. What's more, our results also showed 
279

280

281

282

283

284

285

286

287

288

289

290

291

292

293

294

295

296

297

298

299

300

301

302

303

304

305

306

307

308

309

310

311

312

313

314

315

316

317

318

319

monocytes were significantly lower in patients with radiotherapy than patients without radiotherapy, which may contribute to the resistance of gliomas radiotherapy.

Next, we further investigated tumor-infiltrating macrophages in gliomas. Based on their function, M0 macrophages can be differentiated into two categories: classically activated macrophages (M1 macrophages) and alternatively activated macrophages (M2 macrophages) by stimulation of various cytokines(Chen et al. 2019; Jiang et al. 2019; Lailler et al. 2019). Previous studies show gliomas contain a plenty of macrophages, and tumor-associated macrophages correlate with progression and angiogenesis of gliomas by several ways, including: microRNA-macrophage feedback loop(Bao \& Li 2019; Liu et al. 2019), extracellular lipid loading(Offer et al. 2019) and cytokines like OPN, IRGM, IL-6(HernandezSanMiguel et al. 2019; Wang et al. 2018; Xu et al. 2019). In addition, previous study successfully reeducated the pro-tumor M2 macrophages toward anti-tumor M1 macrophages by a dual-targeting biomimetic treatment strategy which provides a good method for the pharmacotherapy of gliomas(Zhao et al. 2018). Our results showed that M0 and M2 were significantly increased in gliomas, while M1 were significant decreased. The results confirmed pro-tumor function of M0 and M2 as well as anti-tumor function of M1 in gliomas. And the infiltration of M0 macrophages showed correlated with decreased survival $(\mathrm{P}<0.05)$. In addition, we found M0 macrophages were significantly lower in IDH mutant than IDH wildtype. This result may explain glioma patients with IDH-mutant always have favorable survival outcomes. After functional enrichment analysis, 3 gene sets includes 102 genes about angiogenesis were detected in high macrophage-infiltrating group, which was consistent with previous study.

Among 102 core genes, we found 5 stromal signatures correlated with high macrophages infiltration and angiogenesis indicated poor prognosis $(\mathrm{P}<0.05)$. The 5 stromal signatures including HSPG2, FOXF1, KDR, COL3A1, and SRPX2. Sun X. et al constructed the multicellular gene network between gliomas and macrophages, they found macrophage-related gene signature had good prognostic value for predicting resistance to targeted therapeutics and survival of glioma patients( $X$ et al. 2019). And we found the relationship between stromal signatures and macorphages signatures in gliomas. Based on precious studies, high expression of perlecan/HSPG2 in gliomas lead to tumor promotion through the transformation of brain extracellular matrix into tumor microenvironment( $Q$ et al. 2016). In addition, high expression of COL3A1 contributes to glioma cells proliferation and migration( $\mathrm{J}$ et al. 2017). And up-regulation of KDR promotes proangiogenic myeloid cells, result in low-grade to high-grade transition( $Y$ et al. 2017). Moreover, Glioma with high tumor associated macrophages has increased expression of SRPX2(NA et al. 2016). And we also found these five stromal signatures were correlated with clinical features like grades, histology, chemotherapy and radiotherapy. The expression of stromal signatures was significantly higher in patients with higher grade and worse pathological features like glioblastoma. Then, we constructed a prognosis classifier based on HSPG2, FOXF1, KDR, COL3A1, and SRPX2. And the classifier showed strong predictive ability in both CGGA cohort and TCGA cohort. We confirmed the high infiltration of M2 macrophages in protein and histology level via CPTAC and the human protein atlas. Eventually, based on our investigation, up-regulation of 5 stromal signatures may lead to macrophage infiltration and angiogenesis in gliomas patients. And our classifier also provides strong predictive ability in prognosis. Our study may provide a novel immunotherapeutic strategy and a more clearly overview of tumor microenvironment in gliomas.

However, there still exited several limitations in our research. First, the calculation results from public 
320

321

322

323

324

325

326

327

328

329

330

331

332

333

334

335

336

337

338

339

340

341

342

343

344

345

346

347

348

349

350

351

352

353

354

355

356

357

database may show bias. Although we have verified the results in two independent cohorts, we should continue to deeper research. Second, the macrophage infiltration and angiogenesis function of 5 stromal signatures in gliomas need to further confirmation in vitro and in vivo.

\section{Conclusions}

Based on CGGA RNA-seq datasets, the landscape of 22 tumor-infiltrating immune cells in gliomas was analyzed by CIBERSORT. And high infiltration of macrophages was correlated with poor outcome. After functional enrichment analysis, 3 gene sets includes 102 core genes about angiogenesis were detected in high macrophage-infiltrating group. Next we found 5 stromal signatures indicated poor prognosis which including HSPG2, FOXF1, KDR, COL3A1, SRPX2. 5 stromal signatures were adopted to construct a classifier. And the classifier showed powerful predictive ability. Finally, we validated this classifier in TCGA and the result was consistent with CGGA. Our investigation of tumor microenvironment in gliomas may stimulate the new strategy in immunotherapy. And 5 stromal signature correlated with poor prognosis also provide a strong predator of gliomas patient outcome.

\section{Declarations}

\section{Acknowledgements}

We thank Dr.Wang and Dr. Chen from Department of Neurosurgery at Zhujiang Hospital, Southern Medical University for providing us the useful advice.

\section{Availability of data and materials}

The datasets including the clinical information and the gene expression data of glioma patients analyzed during the current study are available in the TCGA database (https://cancergenome.nih.gov/) and CGGA database (http://www.cgga.org.cn/). And the proteomics data of 110 glioma patients from The National Cancer Institute's Clinical Proteomic Tumor Analysis Consortium (CPTAC; https://cptac-dataportal.georgetown.edu/), the histological level research of glioma patients was performed in the human protein atlas (https://www.proteinatlas.org/).

\section{Ethics approval and consent to participate}

Not applicable.

\section{References}

AD G, L V , C K, T V, L B, SW VG, and P A. 2016. Dendritic cell vaccines based on immunogenic cell death elicit danger signals and $T$ cell-driven rejection of high-grade glioma. Science trans/ational medicine 8:328ra327.

AM M, JW T, JK W, and MR W. 2019. Genetic and molecular epidemiology of adult diffuse glioma. Nature reviews Neurology 15:405-417. 
B C, MS K, CL L, AM N, and AA A. 2018. Profiling Tumor Infiltrating Immune Cells with CIBERSORT. Methods in molecular biology (Clifton, NJ) 1711:243-259.

Bao L, and Li X. 2019. MicroRNA-32 targeting PTEN enhances M2 macrophage polarization in the glioma microenvironment and further promotes the progression of glioma. Mol Cell Biochem. 10.1007/s11010019-03571-2

C B, F G, L C, and AF C. 2009. Treg depletion with a low-dose metronomic temozolomide regimen in a rat glioma model. Cancer immunology, immunotherapy : CII 58:1627-1634.

Chen P, Zhao D, Li J, Liang X, Li J, Chang A, Henry VK, Lan Z, Spring DJ, Rao G, Wang YA, and DePinho RA. 2019. Symbiotic Macrophage-Glioma Cell Interactions Reveal Synthetic Lethality in PTEN-Null Glioma. Cancer Cell 35:868-884.e866. 10.1016/j.ccell.2019.05.003

CK L, KM F, K C, CH W, CH H, CS Y, and SF T. 2016. Depletion of B cell CLL/Lymphoma 11B Gene Expression Represses Glioma Cell Growth. Molecular neurobiology 53:3528-3539.

CM J, J C, and M L. 2019. Mechanisms of immunotherapy resistance: lessons from glioblastoma. Nature immunology.

CS C, MD E, and PJ B. 2011. GM-CSF production by glioblastoma cells has a functional role in eosinophil survival, activation, and growth factor production for enhanced tumor cell proliferation. Journal of immunology (Baltimore, Md : 1950) 187:1254-1263.

$E Z, F D, Y M, W H, F L, W M$, and Y Q. 2019. Differences of the immune cell landscape between normal and tumor tissue in human prostate. Clinical \& translational oncology : official publication of the Federation of Spanish Oncology Societies and of the National Cancer Institute of Mexico.

Hernandez-SanMiguel E, Gargini R, Cejalvo T, Segura-Collar B, Nunez-Hervada P, Hortiguela R, Sepulveda-Sanchez JM, Hernandez-Lain A, Perez-Nunez A, Sanz E, and Sanchez-Gomez P. 2019. Ocoxin Modulates Cancer Stem Cells and M2 Macrophage Polarization in Glioblastoma. Oxid Med Cell Longev 2019:9719730. 10.1155/2019/9719730

$H K G, M v d B, A B L, D A R$, and $A M S$ S. 2017. Antibody-drug conjugates in glioblastoma therapy: the right drugs to the right cells. Nature reviews Clinical oncology 14:693-707.

HR A, L C, PD P, F M, and C C. 2016. Patterns of Immune Infiltration in Breast Cancer and Their Clinical Implications: A Gene-Expression-Based Retrospective Study. PLoS medicine 13:e1002194.

J P, AM S, N L, M K, I W, I A, A S, B W, G P, L U, and E T. 2011. Mast cell accumulation in glioblastoma with a potential role for stem cell factor and chemokine CXCL12. PloS one 6:e25222.

J P, T B, PH E, A L, A S, G N, A S, M B, F P, B W, G P, K FN, and E T. 2014. Glioma-derived macrophage migration inhibitory factor (MIF) promotes mast cell recruitment in a STAT5-dependent manner. Molecular oncology 8:50-58.

J R, R I, V V, M K, C T-L, A R, L W, M M, J W, C X, D M, and GD B. 2019. Pathway enrichment analysis and visualization of omics data using g:Profiler, GSEA, Cytoscape and EnrichmentMap. Nature protocols 14:482-517.

J S, HG S, T H, H K, SH K, YS D, SH P, SJ K, and CK P. 2017. Restoration of miR-29b exerts anti-cancer effects on glioblastoma. Cancer cell international 17:104.

Jiang XZ, Deng K, Hua ML, Li Z, and Xu S. 2019. [Promoting migration and apoptosis of glioma cells by macrophage migration inhibitory factor]. Zhonghua Bing Li Xue Za Zhi 48:108-111. 10.3760/cma.j.issn.05295807.2019.02.006

Peer) reviewing PDF | (2019:12:43839:1:0:NEW 15 Mar 2020) 
JR S, K K, and JA B. 2018. Neutrophil-Guided Drug Delivery for Targeting Residual Glioma Cells. Neurosurgery 82:N7-N9.

KI W, KE R, P C, KA K, and PE F. 2018. T-cell Dysfunction in Glioblastoma: Applying a New Framework. Clinical cancer research : an official journal of the American Association for Cancer Research 24:3792-3802.

L V, and SW VG. 2012. Treg infiltration in glioma: a hurdle for antiglioma immunotherapy. Immunotherapy 4:675678.

Lailler C, Louandre C, Morisse MC, Lhossein T, Godin C, Lottin M, Constans JM, Chauffert B, Galmiche A, and Saidak Z. 2019. ERK1/2 signalling regulates the immune microenvironment and macrophage recruitment in glioblastoma. Biosci Rep. 10.1042/bsr20191433

Liu Y, Li X, Zhang Y, Wang H, Rong X, Peng J, He L, and Peng Y. 2019. An miR-340-5p-macrophage feedback loop modulates the progression and tumor microenvironment of glioblastoma multiforme. Oncogene. 10.1038/s41388-019-0952-x

M A, CA B, JA B, D B, H B, JM C, AP D, K D, SS D, JT E, MA H, DP H, LI-T, A K, S L, JC M, JE R, M R, GM R, and G S. 2000. Gene ontology: tool for the unification of biology. The Gene Ontology Consortium. Nature genetics 25:25-29. 10.1038/75556

M L, Y X, C B, and M W. 2018. Current state of immunotherapy for glioblastoma. Nature reviews Clinical oncology 15:422-442.

M P, LA H, DW A, LC K, K B, TV A, E H-K, MT C, P C, and DC H. 2013. Glioma grade is associated with the accumulation and activity of cells bearing M2 monocyte markers. Clinical cancer research : an official journal of the American Association for Cancer Research 19:3776-3786.

ML B, SLN M, ER A, TR M, AM K, and XO B. 2018. Multidimensional communication in the microenvirons of glioblastoma. Nature reviews Neurology 14:482-495.

NA H, RA E, S K, J Z, K W-H, AK W, CM F, A T, JA R, and TL S. 2016. Telomere profiles and tumor-associated macrophages with different immune signatures affect prognosis in glioblastoma. Modern pathology : an official journal of the United States and Canadian Academy of Pathology, Inc 29:212-226.

Offer S, Menard JA, Perez JE, de Oliveira KG, Chandran VI, Johansson MC, Bang-Rudenstam A, Siesjo P, Ebbesson A, Hedenfalk I, Sundgren PC, Darabi A, and Belting M. 2019. Extracellular lipid loading augments hypoxic paracrine signaling and promotes glioma angiogenesis and macrophage infiltration. J Exp Clin Cancer Res 38:241. 10.1186/s13046-019-1228-6

PS, A M, O O, NS B, JT W, D R, N A, B S, and T I. 2003. Cytoscape: a software environment for integrated models of biomolecular interaction networks. Genome research 13:2498-2504. 10.1101/gr.1239303

PY W, DA R, TS A, S P, RD A, JC L, WT C, JJ Z, M G, DM P, JM M, R L, DM OR, K F, L K, M G, GJ L, E P, S K, A M, C P, RG $S$, and JS Y. 2019. A Randomized Double-Blind Placebo-Controlled Phase II Trial of Dendritic Cell Vaccine ICT-107 in Newly Diagnosed Patients with Glioblastoma. Clinical cancer research : an official journal of the American Association for Cancer Research.

Q H, T K, J F, Q Z, T J, J Y, X J, and J C. 2016. Tumor Microenvironment and Angiogenic Blood Vessels Dual-Targeting for Enhanced Anti-Glioma Therapy. ACS applied materials \& interfaces 8:23568-23579.

R M, S S, and VW Y. 2017. T Cell Exhaustion in Glioblastoma: Intricacies of Immune Checkpoints. Trends in immunology 38:104-115.

SMJ M, SAR M, and M P. 2018. Cancers of the Brain and CNS: Global Patterns and Trends in Incidence. Journal of biomedical physics \& engineering 8:151-152.

Peer] reviewing PDF | (2019:12:43839:1:0:NEW 15 Mar 2020) 
Subramanian A, Tamayo P, Mootha VK, Mukherjee S, Ebert BL, Gillette MA, Paulovich A, Pomeroy SL, Golub TR, Lander ES, and Mesirov JP. 2005. Gene set enrichment analysis: A knowledge-based approach for interpreting genome-wide expression profiles. Proceedings of the National Academy of Sciences 102:15545-15550. 10.1073/pnas.0506580102

TF L, K L, Q Z, C W, Y Y, Z C, SJ Y, X L, Y W, M H, N K, YH X, L Z, and X C. 2018. Dendritic cell-mediated delivery of doxorubicin-polyglycerol-nanodiamond composites elicits enhanced anti-cancer immune response in glioblastoma. Biomaterials 181:35-52.

Wang Q, He Z, Huang M, Liu T, Wang Y, Xu H, Duan H, Ma P, Zhang L, Zamvil SS, Hidalgo J, Zhang Z, O'Rourke DM, Dahmane N, Brem S, Mou Y, Gong Y, and Fan Y. 2018. Vascular niche IL-6 induces alternative macrophage activation in glioblastoma through HIF-2alpha. Nat Commun 9:559. 10.1038/s41467-018-03050-0

X L, S W, Y Y, M Z, G Z, and Z H. 2017. The prognostic landscape of tumor-infiltrating immune cell and immunomodulators in lung cancer. Biomedicine \& pharmacotherapy = Biomedecine \& pharmacotherapie 95:55-61.

X S, X L, M X, Y S, and XD Z. 2019. Multicellular gene network analysis identifies a macrophage-related gene signature predictive of therapeutic response and prognosis of gliomas. Journal of translational medicine 17:159. 10.1186/s12967-019-1908-1

Xu Y, Liao C, Liu R, Liu J, Chen Z, Zhao H, Li Z, Chen L, Wu C, Tan H, Liu W, and Li W. 2019. IRGM promotes glioma M2 macrophage polarization through p62/TRAF6/NF-kappaB pathway mediated IL-8 production. Cell Biol Int 43:125-135. 10.1002/cbin.11061

Y H, PR, W H, CH, B C, JH K, E G, R Y, W C, E V, RX, T Z, D P, J X, J H, I M, H P, J B, E H, BS D, SR, D L, and J G. 2017. A proangiogenic signaling axis in myeloid cells promotes malignant progression of glioma. The Journal of clinical investigation 127:1826-1838.

Zhao P, Wang Y, Kang X, Wu A, Yin W, Tang Y, Wang J, Zhang M, Duan Y, and Huang Y. 2018. Dual-targeting biomimetic delivery for anti-glioma activity via remodeling the tumor microenvironment and directing macrophage-mediated immunotherapy. Chem Sci 9:2674-2689. 10.1039/c7sc04853j

Peer] reviewing PDF | (2019:12:43839:1:0:NEW 15 Mar 2020) 
Figure 1

Adaptive and innate immune cells in gliomas.

(A) Adaptive immune cell subtypes in glioma. The fraction of M0 macrophages, M2

macrophages, resting NK cells, plasma cells, CD8 $+\mathrm{T}$ cells is significantly higher in gliomas tissue than normal brain tissue. (B) Innate immune cell subtypes in glioma. The fraction of naïve B cells, resting dendritic cells, Eosinophils, Neutrophils, M1 macrophages, activated mast cells, follicular helper T cells (Tfh), $ү \delta$ T cells and resting CD4+ memory T cells is significantly lower in gliomas tissue than normal brain tissue. ${ }^{*} p<0.05 ; * p<0.01$; $* * * p<0.001$.

A

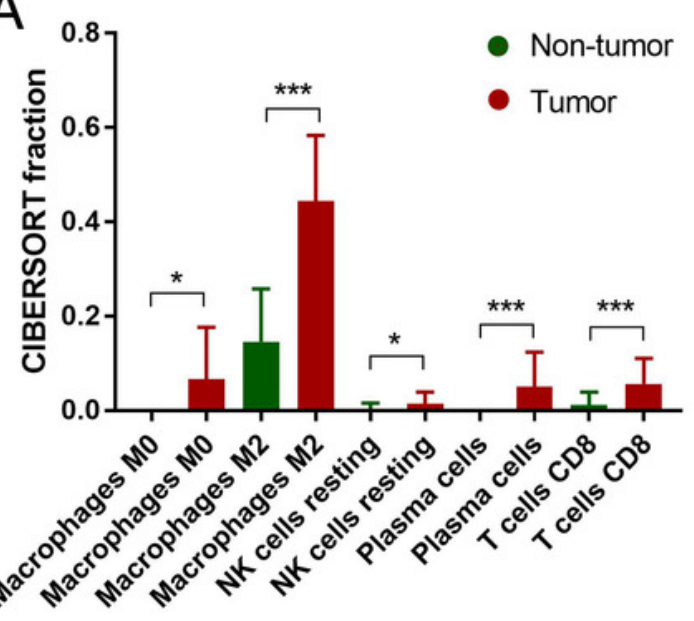

B

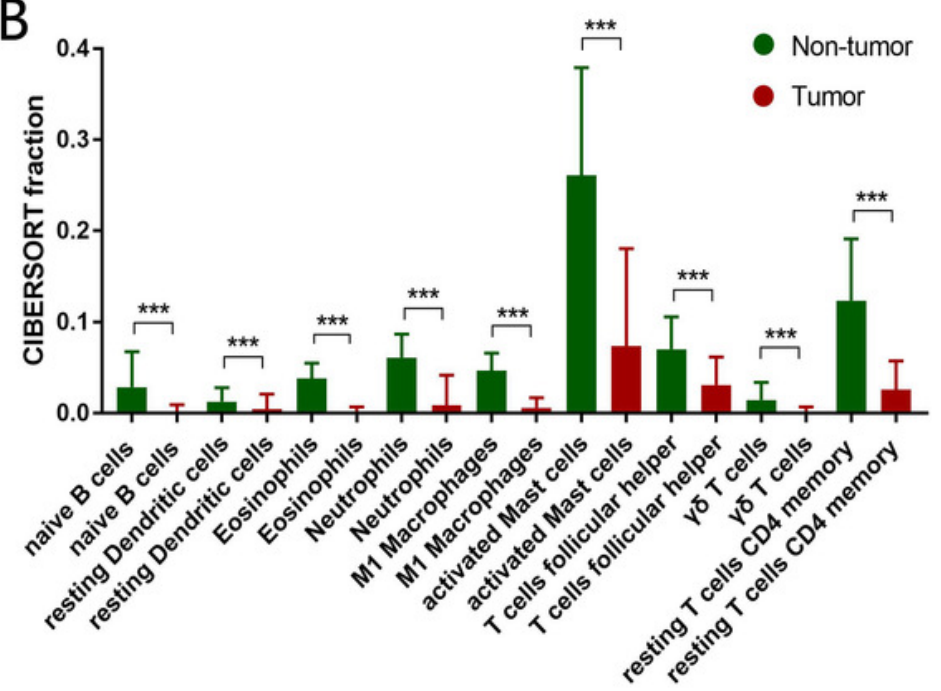




\section{Figure 2}

Correlation between clinical parameters and immune cells.

(A) Monocytes are decreased in elder patients. (B) MO macrophages, Tregs and activated dendritic cells are increased in high grade gliomas, whereas monocytes are decreased. (C)Tregs and MO macrophages are increased in patients with radiotherapy, whereas NK cells and Monocytes are decreased. (C) M0 macrophages, Tregs and $ү \delta$ T cells are increased in glioblastoma (GBM), wherea monocytes and activated mast cells are decreased. (D) Activated mast cells, monocytes and resting CD4+ memory T cells are increased in IDH mutant, whereas M0 macrophages, Tregs, $ү \delta \mathrm{T}$ cells and Tfh are decreased. (E) Tregs are increased in $1 p / 19 q$ co-deletion, whereas naïve $C D 4+T$ cells are decreased. ${ }^{*} p<0.05$; $*^{* * p}<0.01 ; * * * p<0.001$ 
A

Age

- Age $<=44$

- Age $>45$

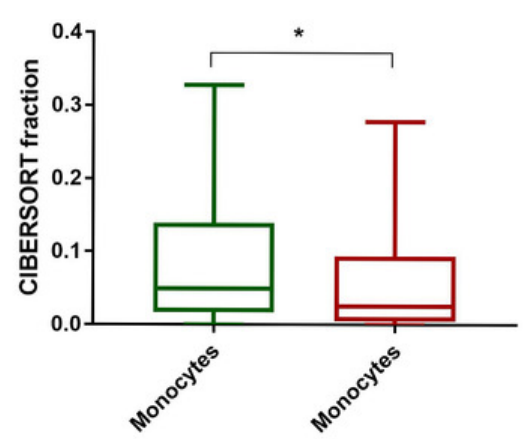

C

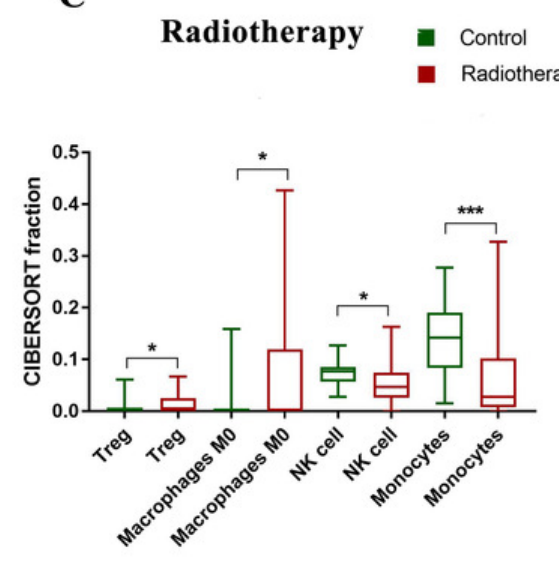

$\mathbf{E}$

1p/19q co-deletion

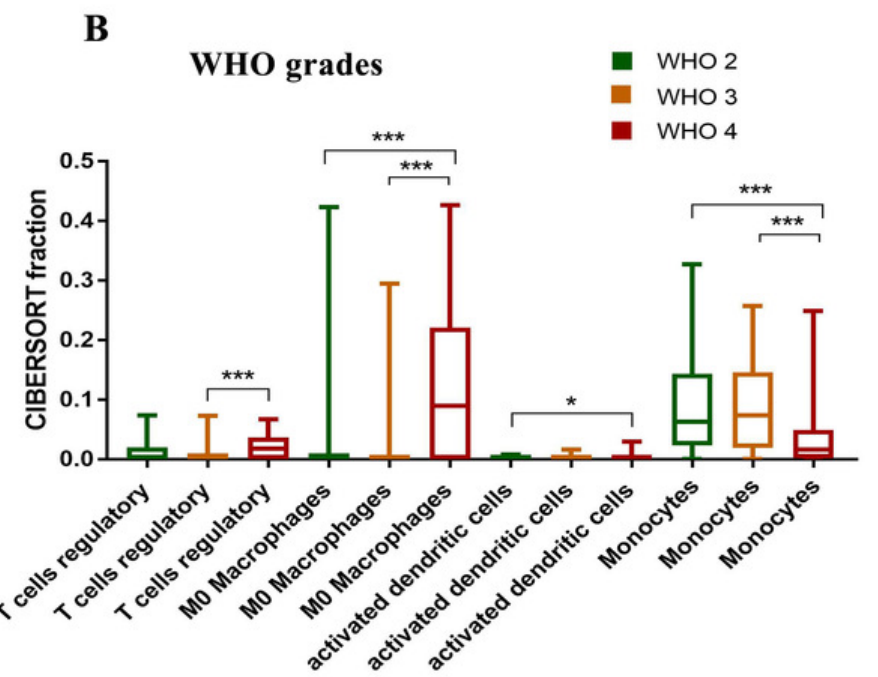

D

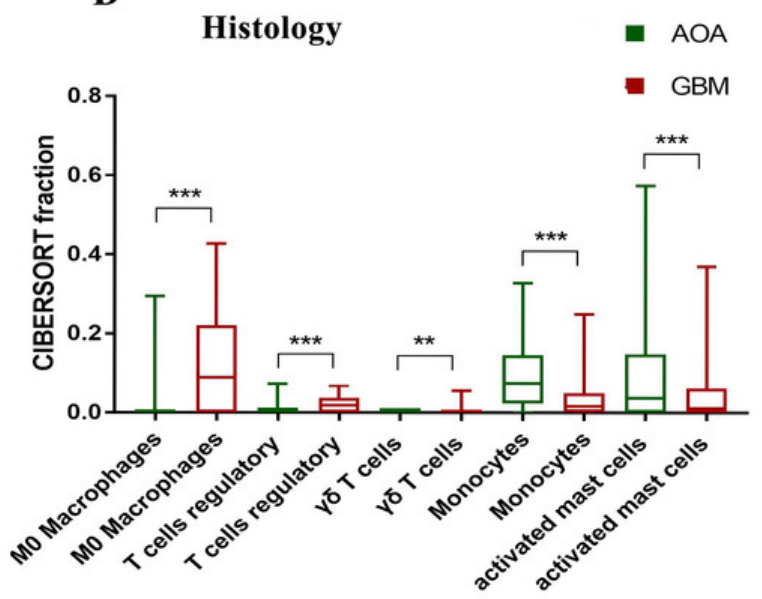

F

IDH mutant

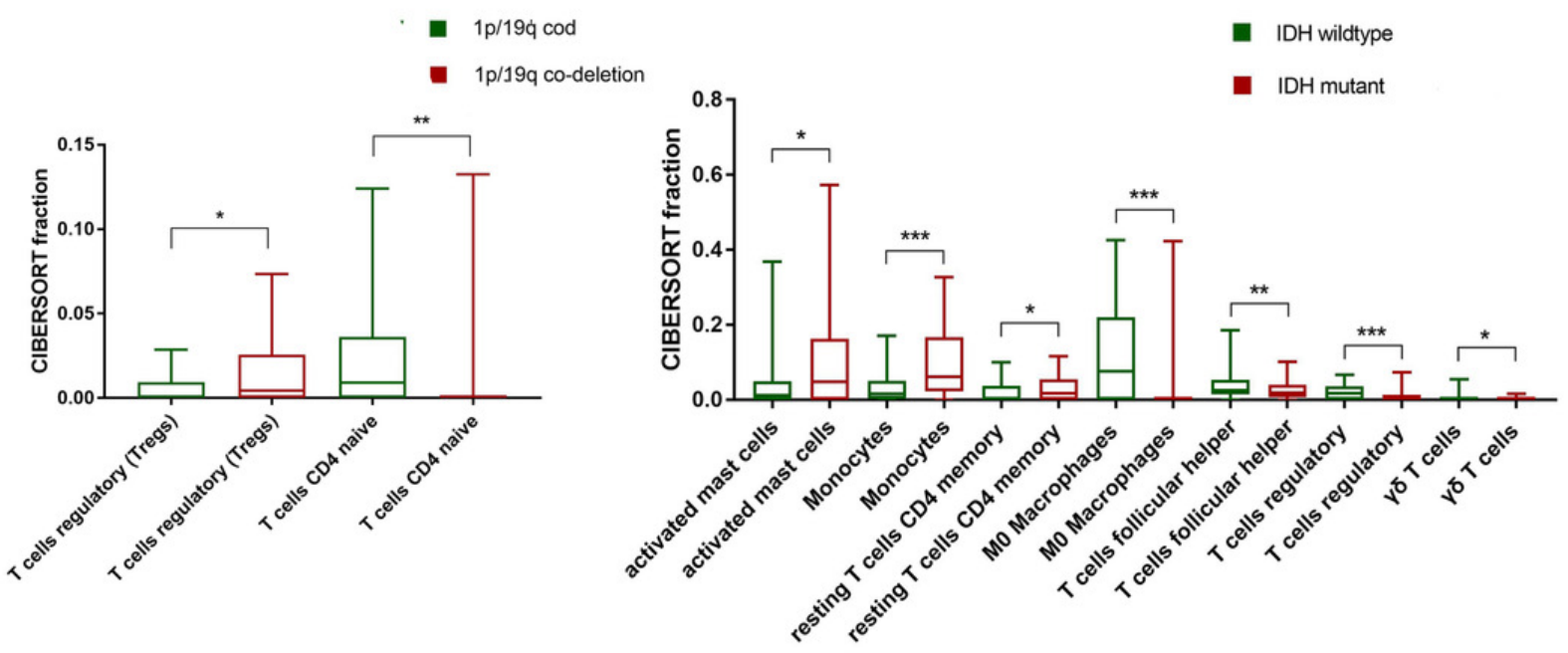


Figure 3

Prognostic value of tumor-infiltrating immune cells in gliomas.

(A) The high infiltration of resting CD4+ memory T cells are correlated with poor outcome $(P=0.027)$. (B) The high infiltration of M0 macrophages are correlated with poor outcome $(P=0.0104)$. (C) High macrophage score are correlated with poor outcome $(P=0.0139)$. 

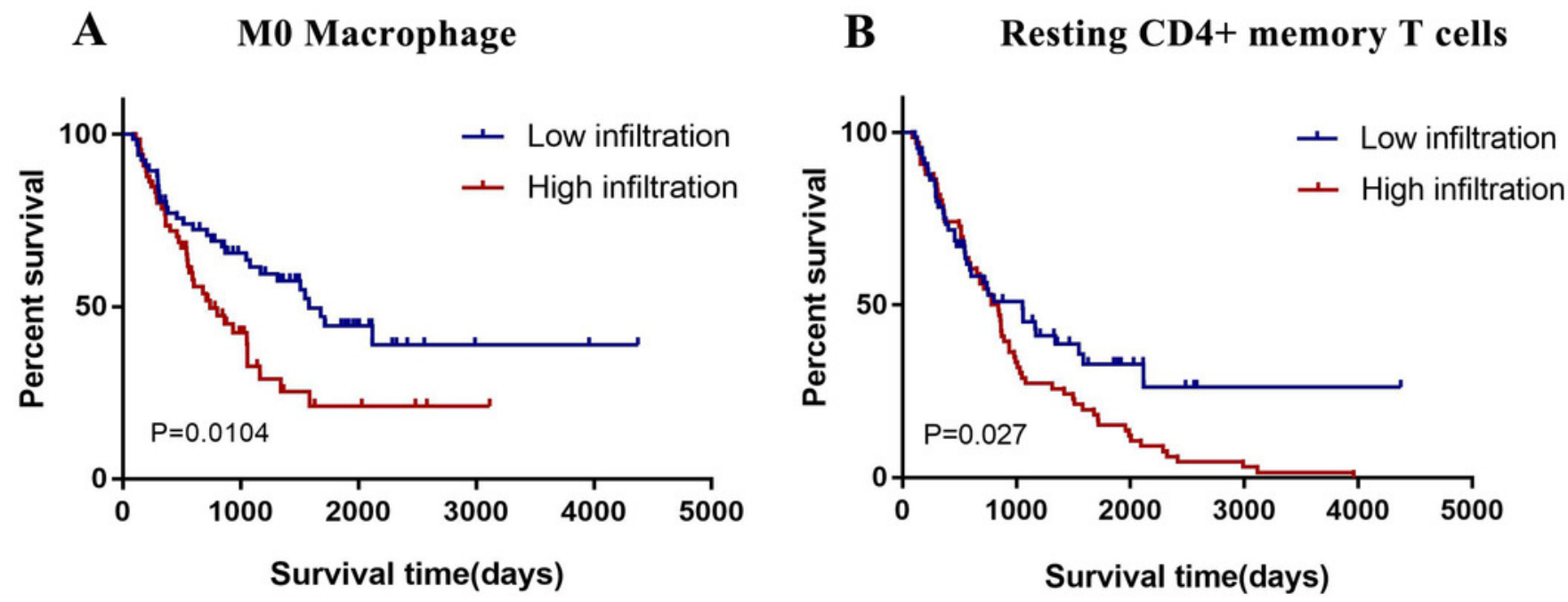

C Macrophage score

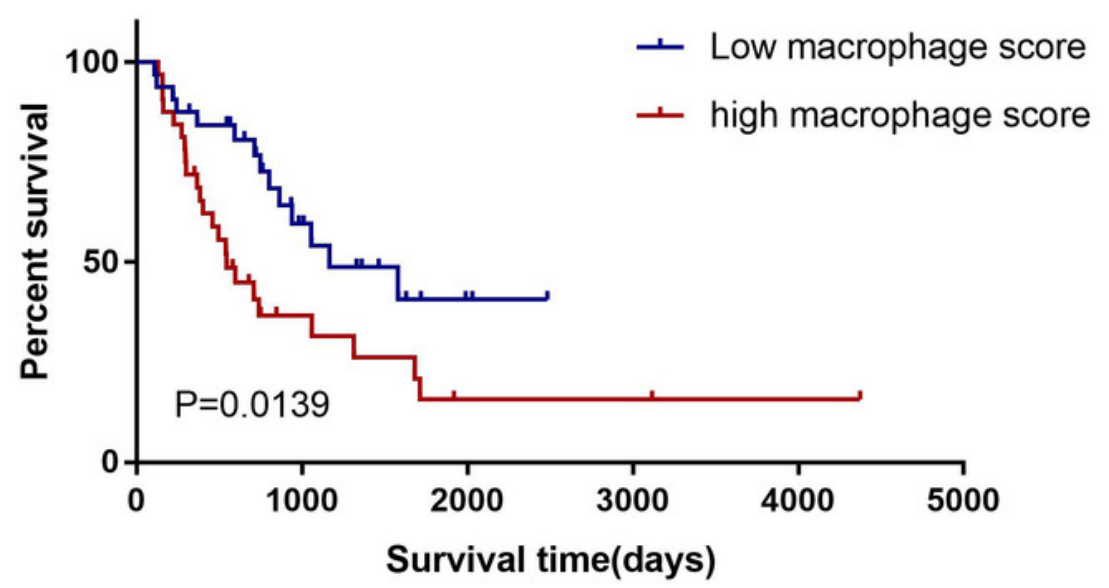




\section{Figure 4}

Functional enrichment analysis of gene signature of macrophages.

GO term and KEGG pathway analysis for the high macrophage-infiltrating group. Pathways with FDR $<0.1 \mathrm{P}<0.01$ are shown. (A) Biological process. (B) Cellular components. (C) Molecular function. (D) KEGG pathway. (E) Enrichment profile generated with GSEA in the gene set "blood vessel morphogenesis" comparing high vs low M0 macrophage-infiltrating group. (F) Enrichment profile generated with GSEA in the gene set "angiogenesis". (G) Enrichment profile generated with GSEA in the gene set "cellular response to vascular endothelial growth factor stimulus". 
A

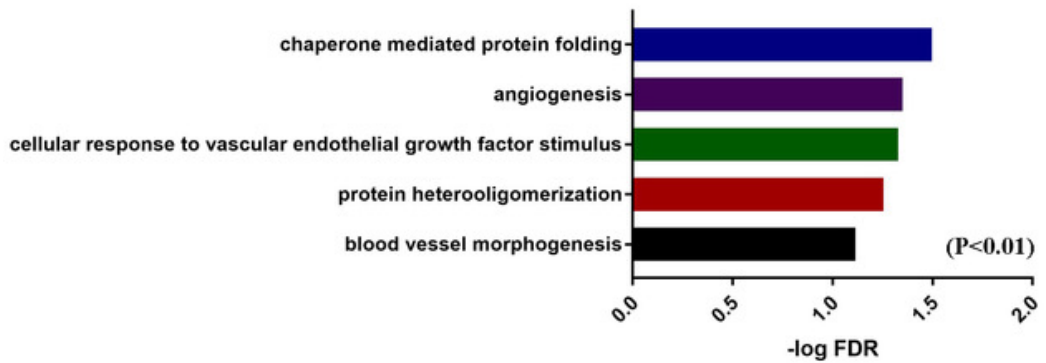

C

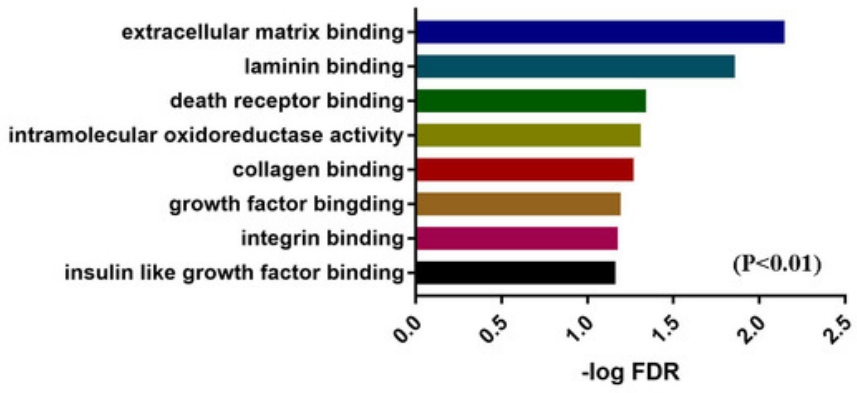

$\mathbf{E}$

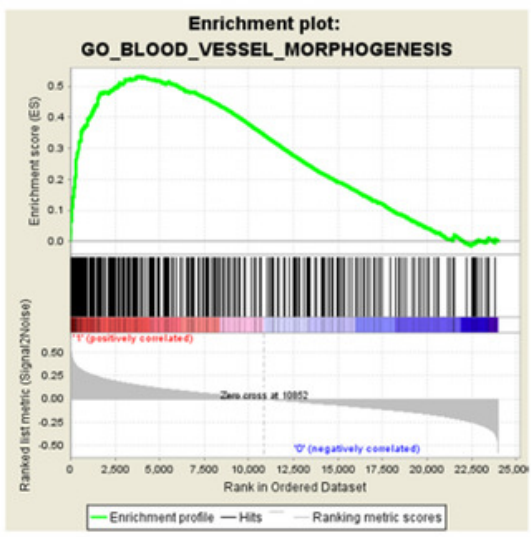

F

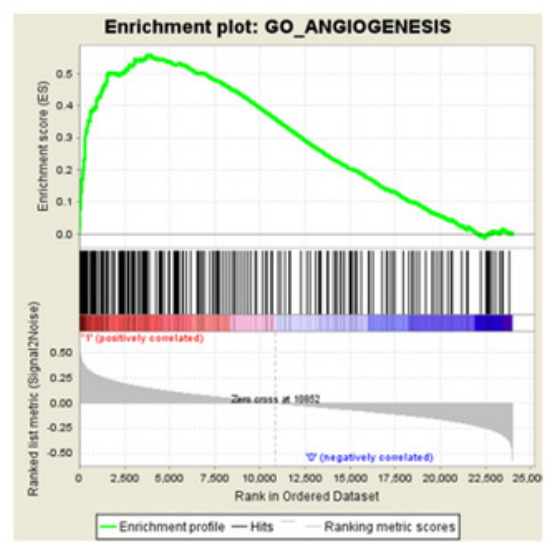

B

cellular components

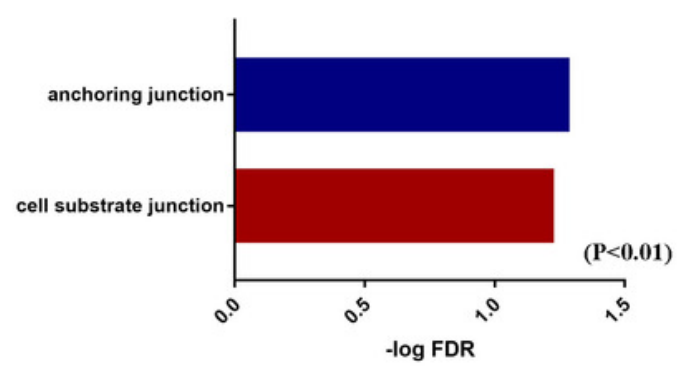

D

KEGG pathway

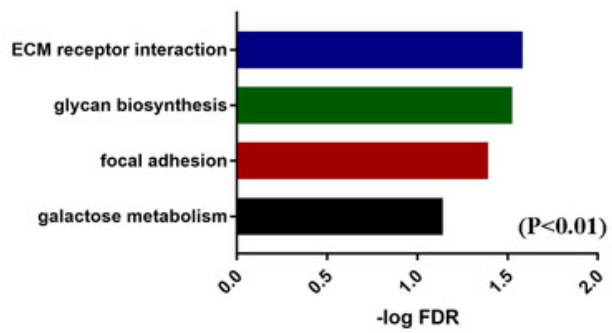

G

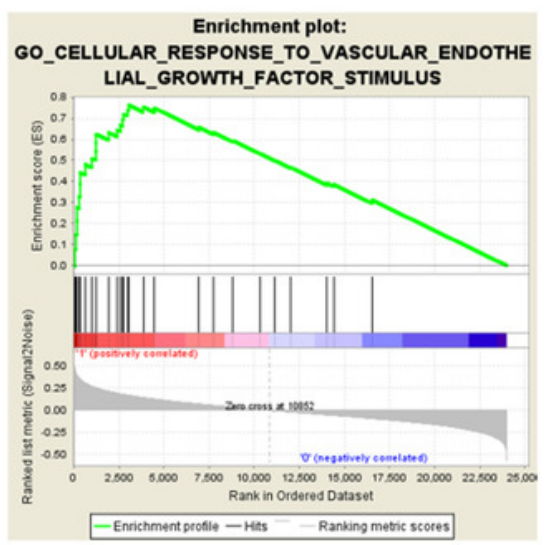




\section{Figure 5}

Investigation of prognostic value of stromal signatures.

(A) Venn diagrams showing the number of commonly genes in hub genes group and stromal signatures. (B, C, D, E, F) The prognostic value of 5 stromal signatures by Kaplan-Meier survival curves in CGGA database. 
A

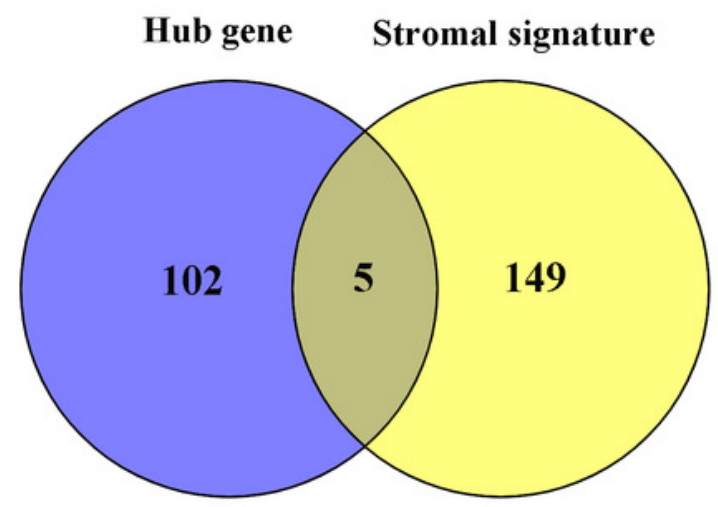

C

FOXF1

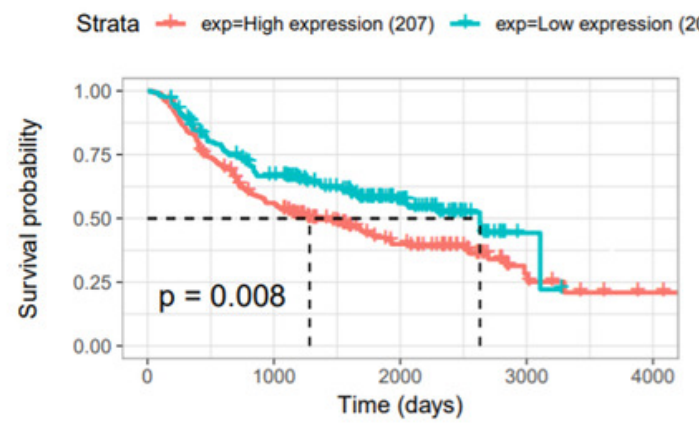

E

\section{COL3A1}

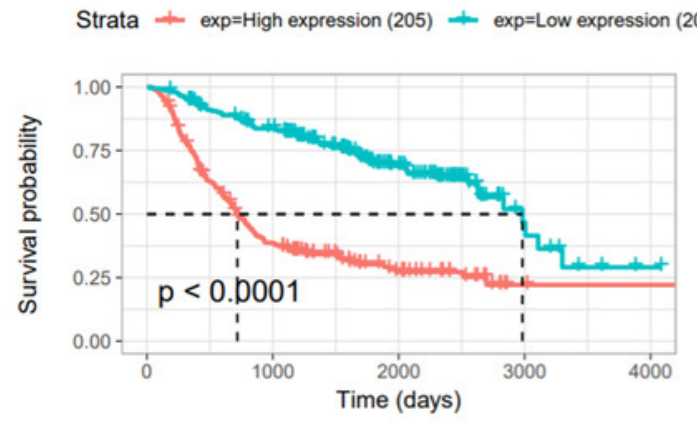

B

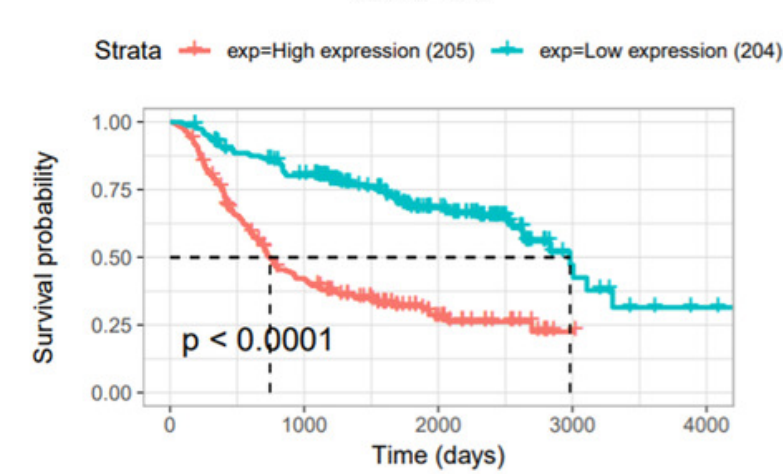

D

\section{KDR}

Strata + exp=High expression (207) + exp=Low expression (202)

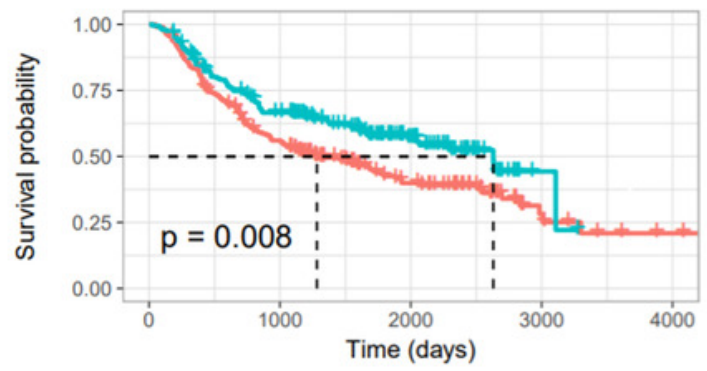

F

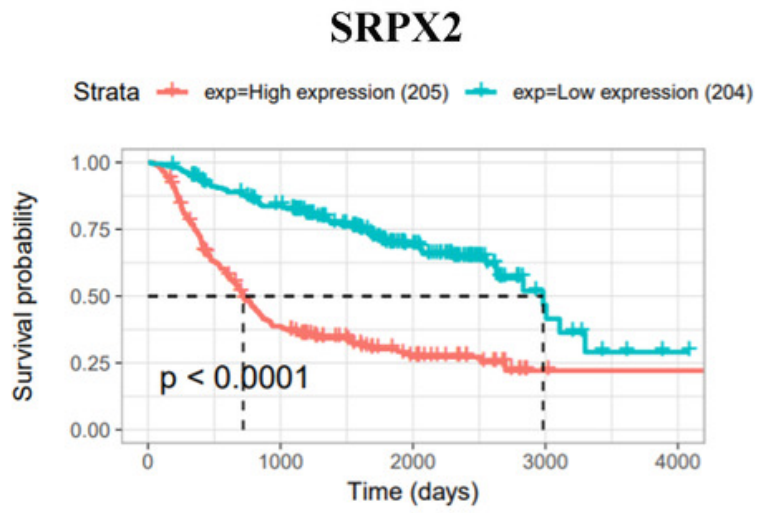




\section{Figure 6}

Construction and verification of prognostic classifier based on stromal signatures.

(A) Forest map based on the univariate COX regressions of 5 stromal signatures. Right vertical dotted line indicates risk genes. (B) Kaplan-Meier survival curves were generated for the comparison of groups of high (red line) and low (blue line) risk score. (C, D) Forest map based on the univariate and multivariate COX regressions which enrolled clinical features and stromal signatures in the overall set. (E) 5 stromal signatures were enrolled in the risk model heatmap. (F) Boxplot shows 5 stromal signatures were correlated with WHO grades. (G) Principal component analysis shows significant different between groups of low (green dots) and high (red dots) risk score. (H) Receiver operating characteristic (ROC) curve plotted to determine the effect of the classifier and compare with other clinical features. (I) The classifier was verified in an independent TCGA cohort. ${ }^{*} p<0.05$; ${ }^{* *} p<0.01 ; * * p<0.001$. 
A

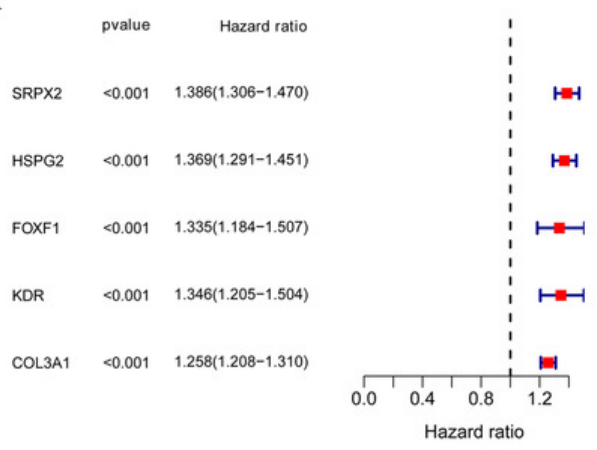

C

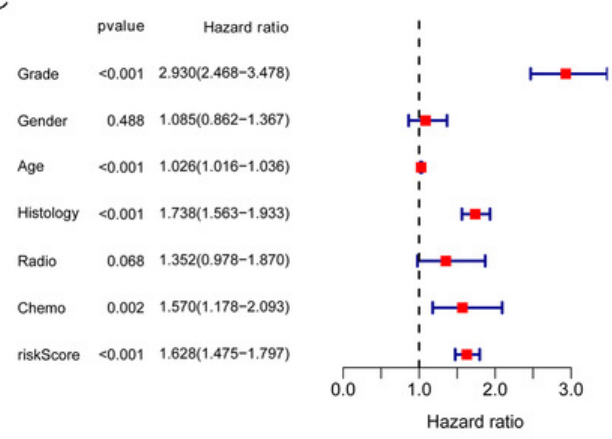

$\mathbf{E}$
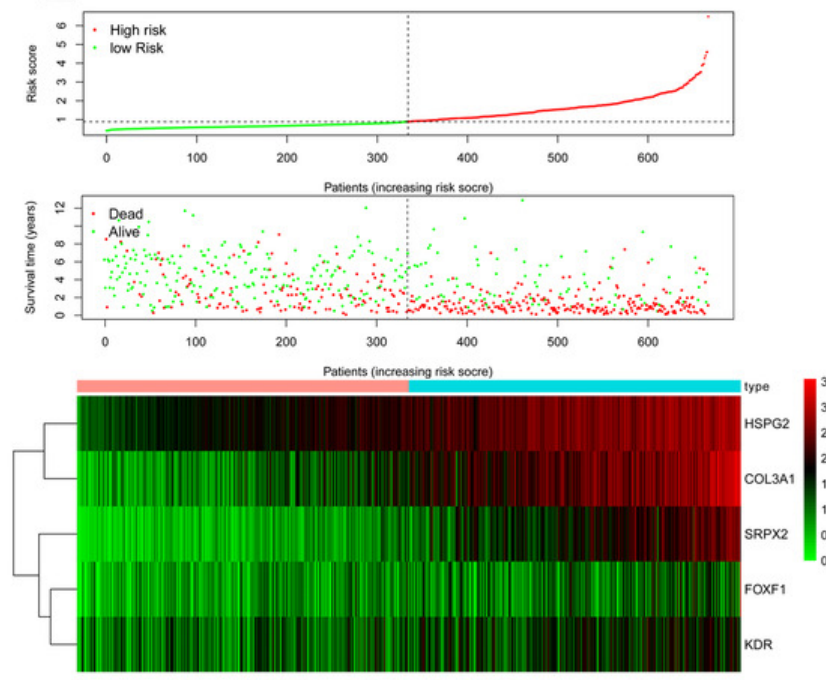

\section{H}

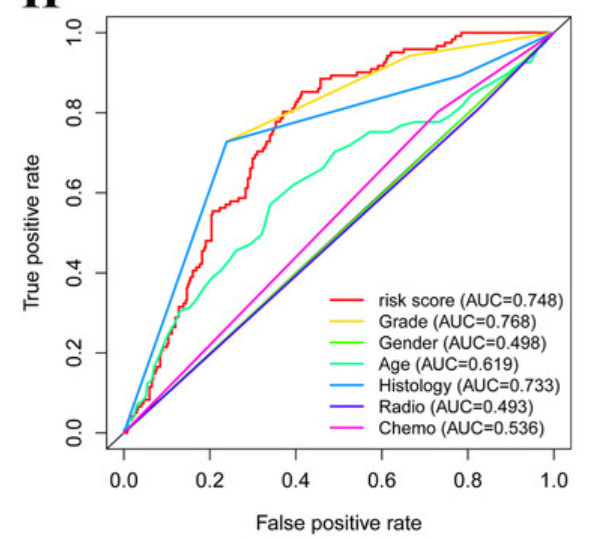

B Risk + High nisk + Low is

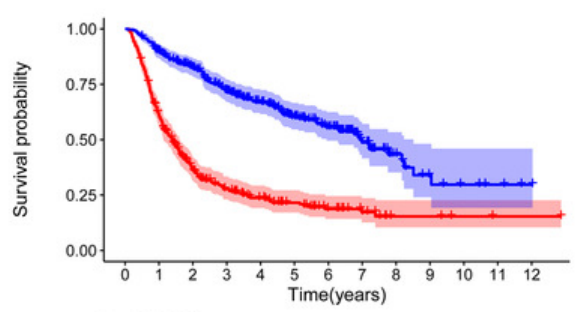

$\mathrm{P}<0.001$

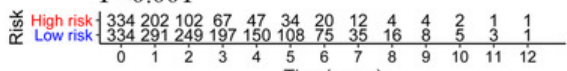

D

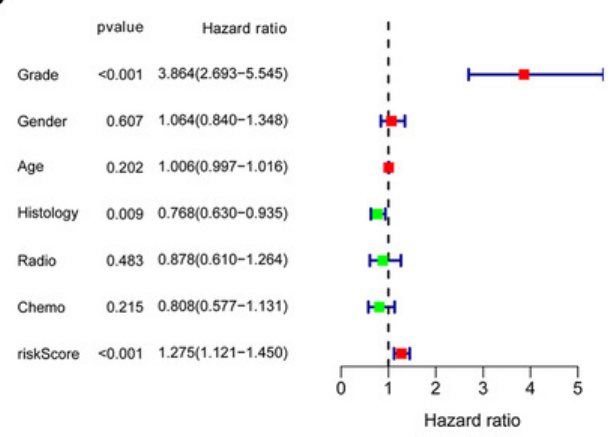

F

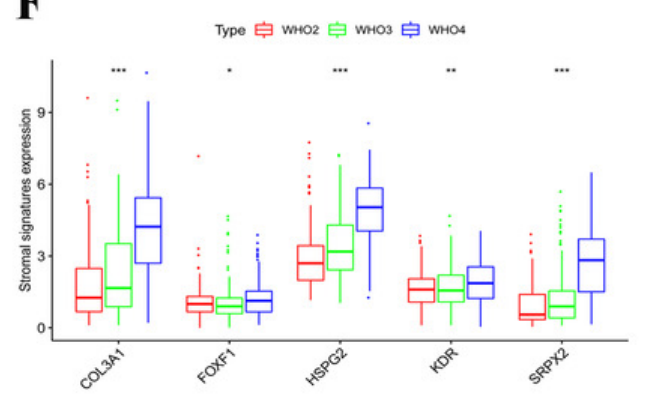

G

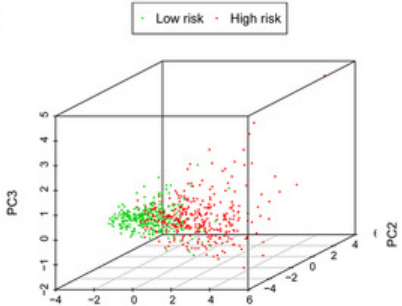

I

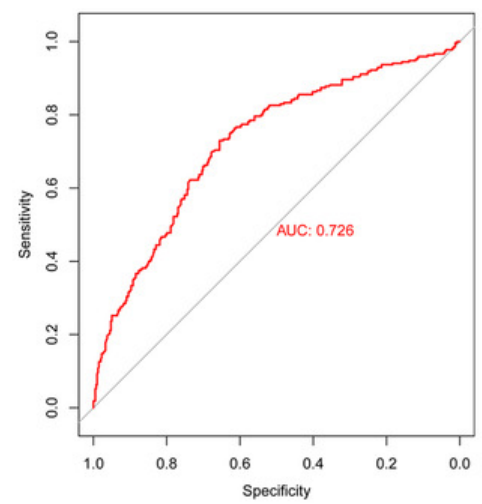




\section{Table $\mathbf{1}$ (on next page)}

Table1. Clinical features of glioma patients in CGGA and TCGA. 
1

\begin{tabular}{ccl}
\hline & \multicolumn{1}{c}{$\begin{array}{c}\text { CGGA } \\
(\mathrm{n}=693)\end{array}$} & \multicolumn{1}{c}{$\begin{array}{c}\text { TCGA } \\
(\mathrm{n}=668)\end{array}$} \\
\hline $\begin{array}{l}\text { Age (year }) \\
>45\end{array}$ & $276(39.8 \%)$ & $337(50.4 \%)$ \\
$\leqslant 45$ & $416(60.0 \%)$ & $331(49.6 \%)$ \\
Gender & & \\
male & $398(57.4 \%)$ & $385(57.6 \%)$ \\
female & $295(42.6 \%)$ & $283(42.4 \%)$ \\
WH0 grade & & \\
WH0 II & $188(27.1 \%)$ & $247(37.0 \%)$ \\
WH0 III & $255(36.8 \%)$ & $261(39.1 \%)$ \\
WH0 IV & $249(35.9 \%)$ & $0(0.0 \%)$ \\
0S (year $)$ & & \\
$\leqslant 1$ & $163(23.5 \%)$ & $244(36.5 \%)$ \\
$1-3$ & $236(34.1 \%)$ & $298(44.6 \%)$ \\
$3-5$ & $111(16.0 \%)$ & $70(10.5 \%)$ \\
$\geqslant 5$ & $110(15.9 \%)$ & $56(8.4 \%)$ \\
\hline
\end{tabular}

